\title{
Dynamic Modeling of Resource Allocation for Project Management in Multi-Project Environment
}

\author{
Mst Taskia KHATUN ${ }^{\text {a, }}{ }^{1}$, Kazuo HIEKATA ${ }^{\text {a }}$, Yutaka TAKAHASHI ${ }^{\mathrm{b}}$ and \\ Issac OKADA $^{\mathrm{c}}$ \\ ${ }^{a}$ Graduate School of Frontier Sciences, The University of Tokyo, Japan \\ ${ }^{b}$ School of Commerce, Senshu University, Japan \\ ${ }^{c}$ Fujitsu Limited, Japan
}

\begin{abstract}
A challenge in project management in a multi-project environment concerns sharing human resources across projects. Generally, project-based organizations share resources to regulate and plan the project portfolio. This resource allocation process can be elevated through the estimation of required resources by assessing different strategies. Two strategies, static and dynamic, have been applied for resource allocation and compared project performance during this work. Our contribution focuses on how a resource-constrained and scheduleconstrained project management policy can meet the planned project performance. Switching resources through dynamic allocation ensures that a project can be delivered on time even when there is no free resource capacity and hiring new staff. Besides, productivity changes as resources are switched among projects back and forth by exploring the gap between the actual and the expected completion dates that affect project performance. Three example projects portfolio has been analyzed employing a system dynamics simulation model. The aim is to plan and control the projects' progress in a multi-project environment by analyzing human resources changes, which will help portfolio managers decide optimal resource allocation.
\end{abstract}

Keywords. Human resource allocation, Multi-project environment, System dynamics, Transdisciplinary

\section{Introduction}

While working in a multi-project environment, project managers generally face problems regarding resource allocation to ensure the timely execution of projects since projects are complex and dynamic. This study focuses on human resource allocation by applying static and dynamic allocation strategies to share resources from a fixed shared resource pool. Considering these resource allocation strategies, we developed a system dynamics (SD) model. This study's primary insight is that projects are unfolding in a multi-project environment frequently switch resources between projects that become a root cause of schedule pressure. Working under schedule pressure and fixed resources shows how resources can be allocated through these allocation strategies to handle project performance.

\footnotetext{
${ }^{1}$ Corresponding Author, Mail:[msttaskia@s.h.k.u-tokyo.ac.jp].
} 
In a multidimensional project environment, projects are often related to each other. Digitization is specified by considering various interfaces and end-to-end processes represented as an integrated and integrative discipline[1]. All procedures must be driven forward, focusing on planning and controlling the entire project environment rather than individual projects. This situation increases the importance of multi-project management (MPM), which focuses on selecting, planning, controlling, and monitoring the entire project environment [1]. Although several projects are handled simultaneously in a multi-project environment, the projects may vary in size, required skill, and different stages of completion but use the resources from the same resource pool [2, p. 1]. Hence, managers responsible for resource allocation often get into difficulties regarding the proper distribution of their resources and confirm the projects' timely execution [3]. A different environmental situation may cause variation in resource management policy in MPM. A common approach for solving resource allocation issues in a multi-project environment in the literature assumes a resource sharing policy among different projects forming a sharing resource pool [4].

This study analyzes human resource allocation strategies in a multi-project environment and figures out how these resource allocation strategies affect project performance. A system dynamics (SD) model has been developed to model human resource allocation in a multi-project environment. We assumed some conditions to obtain project performance through our simulation- first, no free resource capacity; second, no hiring new staff; third, switching resources to the concurrent projects affects productivity.

The contribution of this work is to address resource allocation decision problems with simulation studies. We have compared two different resource allocation strategies and analyzed the resource buffer sizing in a multi-project environment. The simulation results provide necessary decision-making implications for resource allocation strategies, which will help managers form decisions about optimal resource allocation.

We structured the paper as follows. Chapter 1 considers literature reviews that imply the desirable scope of importance of resource allocation strategies for concurrent projects in a multi-project environment. Chapter 2 describes the methodology and the process of application in a multi-project environment. Chapter 3 explored result analysis. Based on the exploration, we made a discussion in chapter 4 . Finally, chapter 5 represents the conclusion.

\section{Literature Review}

\subsection{Multi-project management(MPM)}

Project management is a complex task as it involves complexity, uncertainties, and many activities, even in a single project [5][6]. MPM defines where multiple projects are implemented concurrently, and usually, the projects are lead by one project manager [6][7]. The projects involve in a multi-project environment are often intertwined due to interdependencies among inputs, outputs, and sharing of specialized resources [4][8]. Since multiple projects are unfolded concurrently in a multi-project environment, different issues may arise for successful project execution. Many studies have worked on several matters regarding MPM. These studies have discussed resource allocation issues [3][4][9][10], project scheduling issues [4][9], project delays as a result of 
managerial problems [4][10]. All these studies have focused on organizational design and the management of the projects.

This study focused on resource allocation problems in a multi-project environment. Often sharing the same resources to concurrent projects make the allocation problem complex since it creates interdependencies among projects. Due to these interdependencies, a resource problem in one project may cause a problem for another project. Hence, the proper resource allocation plan is crucial for the better performance of the project organization.

\subsection{Multi-project management and approaches}

For the timely execution of the concurrent projects in a multi-project environment considering challenges of interdependencies, project priority, and resource capacity, the researchers have addressed several approaches. A heuristic operational research approach through optimization algorithms' involvement has been discussed [11][12]. A method regarding resource sharing policies with dedicated \& core teams and shared resource pools has been explained [10][13]. The main focus is dealing with conflicts among project management, resource management, and portfolio management. Multiagent-based decentralized decision-making policy design methods have been developed concerning multi-project environments [9][14].

\subsection{Resource allocation in a multi-project environment}

One principal challenge in a multi-project environment is how to allocate resources to ascertain the timely execution of all planned projects. Usually, to increase the available resource utilization, project managers tend to distribute all the resources at the beginning of the cycle of all planned projects. But this process doesn't focus on uncertainties involved in project execution. A multi-project environment requires a dynamic and efficient way of determining resource allocation and a pragmatic set of delivery schedules for projects, especially when resources need to switch during ongoing projects [15]. Using resources from a shared resource pool and having interdependencies among projects creates difficulties for project managers about how the resources should be allocated and distributed [16]. Several types of research have proposed and discussed methods for resource allocation, focusing on MPM. A bottom-up approach for resource allocation has been defined to minimize duration in the presence of uncertainty [17]. A system dynamics simulation model has been developed to simulate the effects of resources allocated projects which arrived late and contributed to design schedule-driven project management policy[4]. A decision support system has been developed to mitigate resource competition of autonomous local decisions [9].

[4] work is related to our study, but the focus is different. Their work has focused on schedule pressure by considering the delay only for the first projects to obtain planned project milestones in the long-term and contributing to illuminating short-term logic for tardy projects. In our study, we reproduced project behavior by considering the delay for all the concurrent projects as a long-term effect and obtained the outcomes of project execution in the presence of delay and fixed resource capacity. We considered rework and its impact on the project progress mechanism's performance to reproduce project behavior that has not been considered in the prior work. Considering these, we developed an SD model and then analyzed the simulation results to explore project performance. 


\section{Research methodology}

\subsection{Overview of a dynamic model for human resource allocation}

The overview of the proposed methodology has shown (Figure 1). In a multi-project environment, a set of projects run concurrently. Hence, the project manager assigns resources to each project based on its necessity and deadline and switches the resources from one project to another as needed. Tasks are developed for each project concurrently, shown through the causal loop diagram (CLD). The CLD for this work represents the interrelation among the project development process parameters considering a multiproject environment. A system dynamics (SD) simulation model has been developed based on the resource allocation process and causal mapping for task development. After developing the simulation model, several runs have been done to obtain resource allocation and project performance in a multi-project environment.

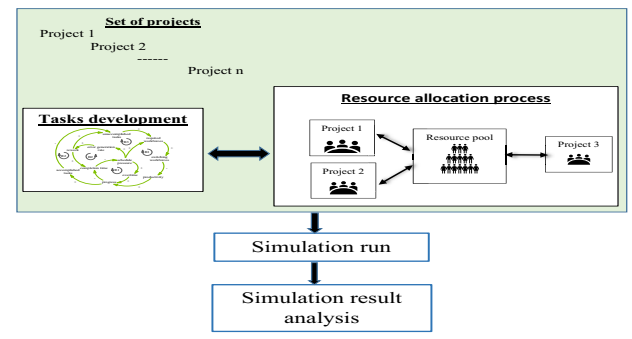

Figure 1. Overview of the proposed methodology.

\subsection{The conceptual model}

\subsubsection{Causal loop diagram (CLD)}

In this section, the process described above is illustrated.

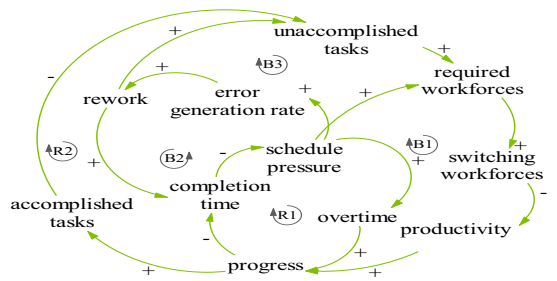

Figure 2. CLD for task accomplishment.

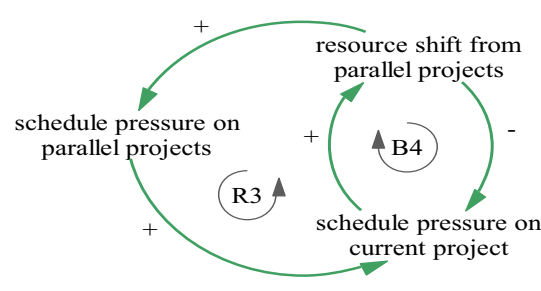

Figure 3. CLD showing the impact of schedule pressure for resource allocation in multi-projects.

Figure 2 depicts the dynamics of effective parameters of project tasks development and resource allocation regarding various reinforcing and balancing loops represented as causal loop diagram (CLD). A CLD in system dynamics is used to model complex relationships with feedback among different parameters involved. In the CLD, the $(+)$ sign represents that both variables are affected in the same way; the (-) sign defines both variables are affected in a reverse manner. The overall reinforcing loop is denoted with ' $\mathbf{R}$,' and the balancing loop is indicated with 'B.' In figure 2, the reinforcing loop R1 shows how progress is affected by schedule pressure and affects completion time. Another reinforcing loop, R2, shows the relationship between project task accomplishment and the required workforces, which causes workforces switching and 
affects productivity. For balancing loops, B1 shows the relation between schedule pressure and its impact on workforces, and loop B2 shows the effect of schedule pressure on rework and completion time. Another balancing loop, B3, represents rework with unaccomplished tasks, which eventually affects project completion time. While talking about schedule pressure, the loops R3 and B4 in figure 3 shows how the schedule pressure is affected due to resource switching.

\subsubsection{Project progress mechanism and resource allocation}

Figure 4 is used as a project progress mechanism and shown as a stock-flow diagram to determine the project progress rate and track project task accomplishment. Each project consists of tasks referred to as original work to do that needed to be accomplished for project execution. When the tasks are completed, they are referred to as work done. This work done is measured through the flow progress rate. When tasks begin to develop, it contains an error generated through the flow rework generation and then initially moves to the stock undiscovered rework. Rework is an error-based task that is needed to be developed again to complete project execution. After stored in the stock undiscovered rework, rework is discovered with time through the flow rework discovery and then proceeds to another stock rework to do. Rework to do increases the number of unaccomplished tasks and requires more time to accomplish.

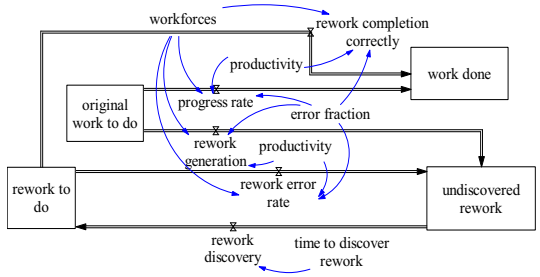

Figure 4. Project progress mechanism.

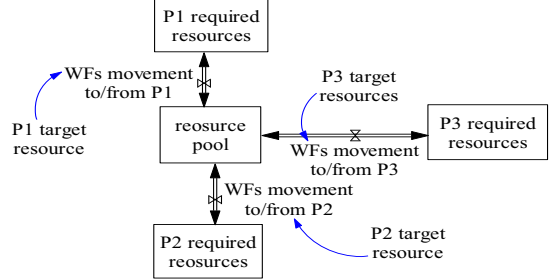

Figure 5. Stock-flow diagram of the resource allocation process.

Resource allocation to several projects in a multi-project environment has been shown as a stock-flow diagram (Figure 5). Once a project arrives, the project manager will capture resources from the concurrent projects considering the gap between the actual and the expected completion date of the concurrent projects. The movement of the resources to the projects will be done through the shared resource pool.

\subsubsection{Model Boundary}

The model boundary considered for the developed model of this work is shown (Figure 6). The changes of these boundary variables have a significant impact on model behavior as well as project performance. Since the simulation model is developed based on several assumptions, the model's endogenous and exogenous variables are defined and then performed the sensitivity analysis by changing the boundary assumptions to obtain the model behavior.

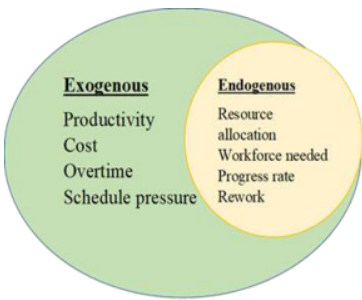

Figure 6. Model boundary. 


\subsubsection{Simulation results analysis}

We simulated three different projects to reproduce a multi-project environment and obtained the performance through project progress, completion time, and resource allocation by switching resources between projects. Based on the causal loop diagram, project progress mechanism, and resource allocation process, we developed the simulation model using the Vensim tool and then run and analyze the simulation results.

\section{Simulation results and analysis}

\subsection{Resource allocation strategies}

Project development in a multi-project environment often experiences several challenges that might be attained through contextual factors-resource availability, project scope, time, client expectations, etc. In regards to these, resource allocation processes are impacted by different strategies. Considering projects' deadlines and required resources, two strategies have been applied and discussed in this work.

\subsubsection{Dynamic resource allocation strategy}

Dynamic resource allocation allows allocating the resources to projects dynamically by switching them among projects and keeping down completion time and cost [18]. Since in a multi-projects environment, several projects happen concurrently, the projects can be started any time after getting approval. While assessing a dynamic resource allocation strategy, we considered two different situations of the projects beginning.

i. A gap between the projects (considering delay): In this case, a delay has been considered while starting the projects. During the simulation run, we have considered delays for project 2 and project 3. Project 1 has been started at the due time.

ii. All projects start simultaneously (without delay): Projects will start simultaneously, and the resources will be distributed at the beginning based on the requirements.

\subsubsection{Static resource allocation}

In this allocation process, each project is developed separately as a single project environment. After finishing one project, the next project will begin.

\subsection{Project data}

Table 1. Input parameters for simulation run.

\begin{tabular}{cc}
\hline Parameters & Input values \\
\hline Number of projects & 3 \\
Project scope & 200 tasks (for each) \\
Resources & 30 persons \\
Nominal productivity & 0.05 task/person/day \\
\hline
\end{tabular}


As previously mentioned, three projects have been considered to analyze the resource allocation process and its impact. For each project, the scope is assumed to the same. Therefore, the initial run of the model was conducted under the initial conditions (Table 1).

\subsection{Results analysis}

\subsubsection{Dynamic resource allocation (considering gap)}

Focusing on this state while simulating the designed projects to reproduce a multi-project environment, a gap has been considered, representing the project's delay. This system's situation often increases schedule pressure on the project and becomes a reason for the other projects' resource shifting.

As mentioned, the delay has been considered for project 2 and project 3 . Hence when project 1 begins, all the resources are allocated to project 1 , as they are all available at that time. On the other hand, when project 2 schedules to unfold concurrently with project 1 after a certain period, it captures some of the resources from project 1 (Figure 7) but didn't get the desired number of resources since project 1 still ongoing. These situations increase schedule pressure both for project 1 and project 2. To tackle these circumstances, workforces need to do overtime at times, keeping the deadline.

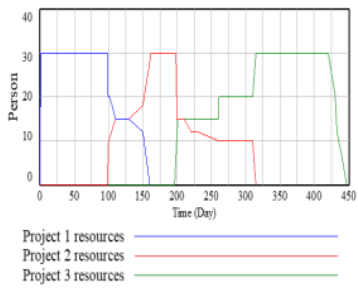

Figure 7. Resource allocation to each project.

In a later stage of the simulation, the beginning of project 3 captures resources from project 2 and creates the same situations as previous projects. In the meantime, project 1 execution process is completed, and the resources are allocated to other projects. This dynamic behavior of resource allocation simulates that the management process has no alternative but to capture resources from other projects and happen concurrently. Switching resources to and from the concurrent projects are happened by calculating the gap between the actual and the expected completion time. This circumstance causes the shifting of the resources several times and occurs oscillation in resource allocation (Figure 7). The shifting of resources from one project to another negatively affects productivity. This effect on productivity increases schedule pressure and affects the overall progress of the projects. Figure 8 and figure 9 represent the project progress rate and the cumulative progress for each project.

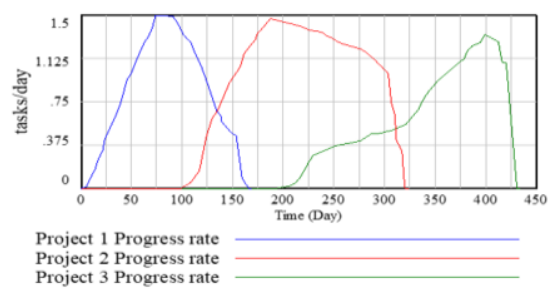

Figure 8. Projects' progress rate.

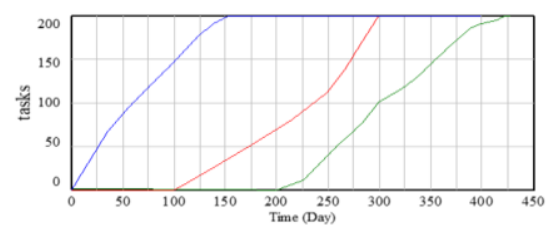

Project 1 task accomplished Project 2 task accomplished Project 3 task accomplished

Figure 9. Cumulative progress of each project.

\subsubsection{Dynamic resource allocation (projects starting at the same time)}

In the multi-project environment, when all the projects begin to unfold simultaneously, resources are distributed to all projects as per requirements. Since in this work, it is 
assumed that all the projects' scope is equal, then resources are distributed equally to all projects. As the resource capacity is fixed, projects often don't get the required resources following the deadline, and workforces need to do high overtime for project completion in time (Figure 10). Since we considered equal project scope for each project, we obtained the same performance for each project (Figure 11).

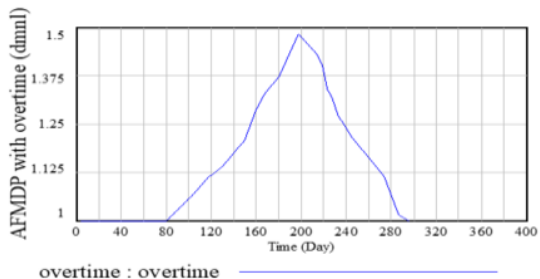

Figure 10. Overtime impact.

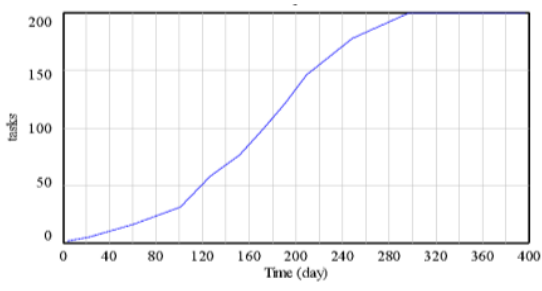

Figure 11. Cumulative progress of each project without delay.

\subsubsection{Static resource allocation}

In this allocation strategy, projects are assumed to be unfolded one after another. This environment usually works as single project development and can be effective if the completion time is flexible and high. In this process, since projects happen singularly, the project manager allocates all the resources to one project until completion. This effect reduces the schedule pressure and makes the project execution process easier (Figure 12).

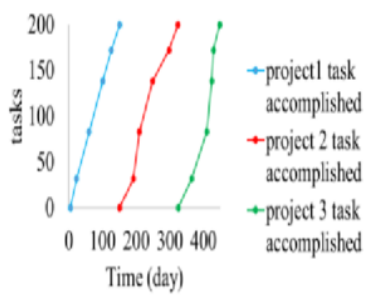

Figure 12. Cumulative progress of each project.

Since multiple projects need to be handled in a multi-project environment and the elapsed time for each project has to be considered seriously, a static allocation strategy cannot be an excellent way to implement this environment.

The simulation results have been explained both for dynamic allocation with delay and static allocation. The obtained resource allocation results act as a helpful paradigm to access the interaction among variables involved in project performance and the effects of managerial decisions for resource allocation in a resource-constrained and scheduleconstrained multi-project environment.

\section{Discussion}

This work exhibits an approach of resource allocation for a multi-project environment sustained on switching resources to concurrent projects with dynamic allocation, affecting the multi-project environment's potential to accomplish planned project milestones. This outcome is beneficial where a fixed shared resource pool is used, and the resource capacity for each project is designated from that fixed shared resource pool. This work explores that several factors happened when the project manager emphasizes completing all projects on time. When one project losses resources, schedule pressure increases for that project. And when several projects have schedule pressure simultaneously, resources switch back and forth in response to the project deadline's 
priority and thus affect productivity. These dynamic allocation consequences operate the schedule pressure in a reinforcing loop yielding a sensible state to deliver the project successfully. The results obtained from the analysis are consistent with [4], where it has also mentioned schedule-pressure is self-reinforcing. With the simulation results, our central contribution shows that the projects developing in a multi-project environment can be delivered on time considering delay and fixed resource capacity. The dynamic allocation process works as a better strategy against total completion time. The first option- allocation considering gap- performs better than the other option. In this case, since a delay happened, so by this meantime, the maximum number of resources can be allocated to the ongoing project, and a large number of tasks can be accomplished before switching resources with high progress. The arrival of new projects causes resource switching and increases schedule pressure. In this allocation process, one issue occurs to the productivity of the resources. When the resources are switched from one project to another, it adversely affects productivity since the project goal is different. The second option of dynamic allocation, when all the projects start simultaneously, requires a longer completion time. The reason for taking a long time is the fixed resource capacity, and at the same time, all projects are active. Hence resources must be distributed to all the projects from the beginning, allocating fewer resources, but often the productivity remains high since switching doesn't happen frequently. They need to do overtime to lower the schedule pressure as well as complete the project. Equality among projects and resource availability are identified as high-performing portfolios in MPM [19]. Hence when the resource capacity is fixed and not having enough availability for each project, the understanding of dynamic allocation could be an excellent way to handle the project execution process, which has been obtained through this study. Another contribution involves experimenting with two different resource allocation strategies separately, explaining how the resources can be handled in different situations and achieve the project milestone without having the worst effect. Another consequence of this work is that the SD modeling has been done based on the rework cycle (Figure 4). The rework cycle focuses on downstream work through which error-based tasks are identified. The project execution process is completed counting all unaccomplished tasks that cover the testing phase of a project.

Any line of employment relies heavily on human resources. While working in a multi-project environment, each project has a distinct aim that necessitates a different set of skills. As a result, the human resources in a multi-project scenario are knowledgeable with a wide range of capabilities. Hence, this study will have a significant impact on the field of transdisciplinary engineering.

\section{Conclusion}

This paper presents an approach for a multi-project environment where each project's resource capacity is determined from the fixed shared resource pool. The resources are eventually shared among projects based on necessity. An essential aspect of this work lies in its usefulness to the decision-makers at various managerial levels while deciding on resource allocation in a multi-project environment. The application of both static and dynamic allocation processes has been explained clearly. Based on the simulation results and overall contribution of this study, it can be said that the simulation provides a stylish representation of the flow of resources in a multi-project environment. In making visible to project managers how they can best meet the resource concerns, this study might 
contribute to decision-making for resource allocation to reduce schedule pressure and improve project performance.

\section{Acknowledgment}

This research work is jointly done with Fujitsu Limited, Japan.

\section{References}

[1] N.N., Multi-project Management, Passcon Consulting Solutions with Passion, 2018.

[2] C. B. Jørgensen, Human resource allocation practices in multi-project organizations: A case study of human resource allocation practices and the contextual conditions that shape them, Master's thesis, University of Agder, Norway, 2015.

[3] T. Ben-Zvi and T. G. Lechler, Resource allocation in multi-project environments: Planning vs. execution strategies, Portland International Center for Management of Engineering and Technology, Portland, OR, 2011, pp. 1-7.

[4] K. Yaghootkar and N. Gil, The effects of schedule-driven project management in multi-project environments, International Journal of Project Management, 2012, Vol. 30, pp. 127-140.

[5] C.M.d.M. Mota, A.T. d. Almeida, and L.H. Alencar, A multiple criteria decision model for assigning priorities to activities in project management, International Journal of Project Management, 2009, Vol. 27, pp. 175-181.

[6] M.C.J. Caniëls and R.J.J.M. Bakens, The effects of Project Management Information Systems on decision making in a multi project environment, International Journal of Project Management, 2012, Vol. 30, pp.162-175.

[7] P. Patanakul and D. Milosevic, The effectiveness in managing a group of multiple projects: factors of influence and measurement criteria, International Journal of Project Management, 2009, Vol. 27, pp. 216-233.

[8] M.A. Kaulio, Project leadership in multi-project settings: Findings from a critical incident study, International Journal of Project Management, 2008, Vol. 26, pp. 338-347.

[9] F. Li, Z. Xu, and H. Li, A multi-agent based cooperative approach to decentralized multi-project scheduling and resource allocation, Computers \& Industrial Engineering, 2021, Vol. 151, 106961.

[10] Z. Laslo and A.I. Goldberg, Resource allocation under uncertainty in a multi-project matrix environment: Is organizational conflict inevitable?, International Journal of Project Management, 2008, Vol. 26, pp. 773-788.

[11] T.R. Browning and A.A.Yassine, Resource-constrained multi-project scheduling: Priority rule performance revisited, International Journal of Production Economics, 2010, Vol. 126, pp. 212-228.

[12] J. Xu and C. Feng, Multimode Resource-Constrained Multiple Project Scheduling Problem under Fuzzy Random Environment and Its Application to a Large Scale Hydropower Construction Project, The Scientific World Journal, 2014, 463692.

[13] H. Chen, G. Ding, J. Zhang, and S. Qin, Research on priority rules for the stochastic resource constrained multi- project scheduling problem with new project arrival, Computers \& Industrial Engineering, 2019, Vol. 137, 106060.

[14] S. Adhau, M.L. Mittal and A. Mittal, A multi-agent system for distributed multi-project scheduling: An auction-based negotiation approach, Engineering Applications of Artificial Intelligence, 2012, Vol. 25, pp. $1738-1751$.

[15] L.D. Dye and J. S. Pennypacker, Project Portfolio Management and Managing Multiple Projects:Two Sides of the Same Coin?, Proceeding of the Project management institute Annual seminars \& Symposium, Houston, 2000, pp. 7-16.

[16] Blomquist, T., \& Müller, R., Practices, Roles, and Responsibilities of Middle Managers in Program and Portfolio Management, Project Management Journal, 2006, Vol. 37, pp. 52-66.

[17] L.D. Long and A. Ohsato, Fuzzy critical chain method for project scheduling under resource constraints and uncertainty, International Journal of Project Management, 2008, Vol. 26, pp. 688-698.

[18] T. Linnenberg, C. Derksen, A. Fay, and R. Unland, Cross-Domain Energy Savings by Means of Unified Energy Agents, Emerging Applications of Software Agents in Industry, 2015, pp. 247-268.

[19] R.G. Cooper, S.J. Edgett, and E.J. Kleinschmidt, Benchmarking Best NPD Practices-II, Research Technology Management, 2004, Vol. 47, pp. 50-59. 